\title{
Bedside ultrasonography-Applications in critical care: Part II
}

\author{
Jose Chacko, Gagan Brar
}

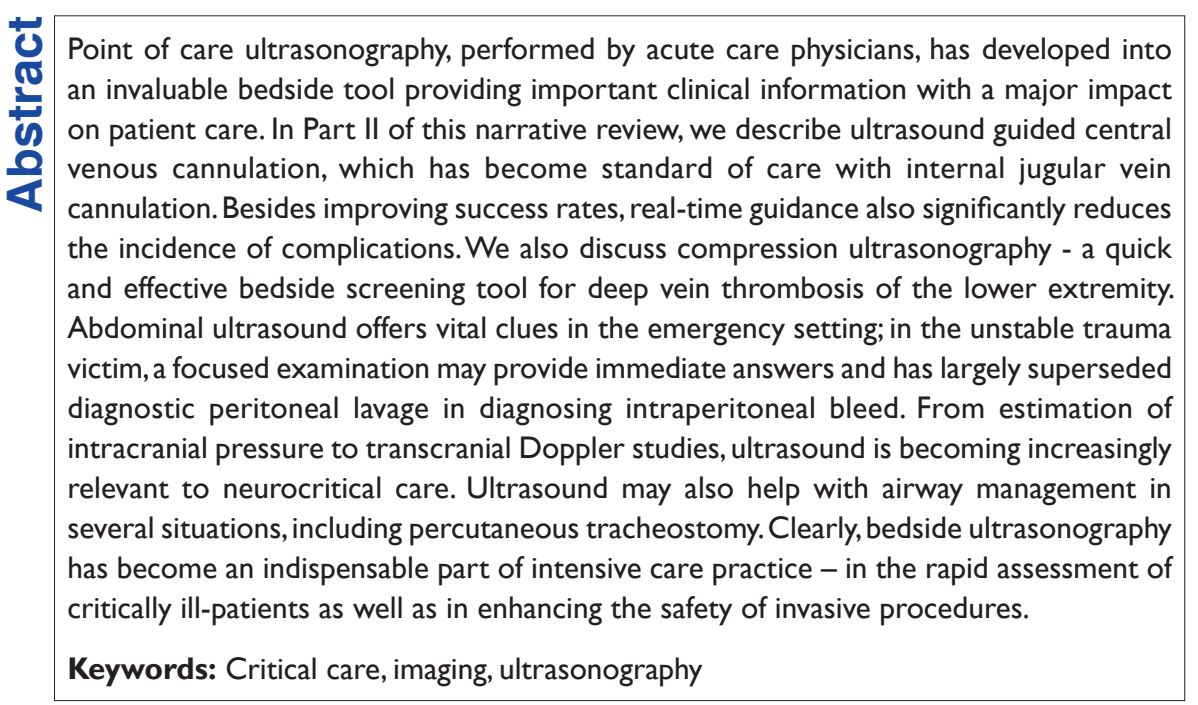

\begin{tabular}{|l|}
\hline Access this article online \\
\hline Website: www.ijccm.org \\
\hline DOI: 10.4103/0972-5229.133897 \\
\hline Quick Response Code: \\
\hline
\end{tabular}

\section{Introduction}

Several innovative applications have emerged from the use of ultrasonography in the acute care setting. Intensive care physicians have taken the lead in discovering novel uses for a technology that has been in use for more than half a century. The inherent safety and relative ease of learning as well as the availability of portable machines that offer high quality images have all led to acute care physicians embracing this technique with enthusiasm. In Part II of this review, we describe the applications of ultrasound for vascular access, diagnosis of deep vein thrombosis (DVT), imaging of the abdomen, nervous system, and the airway.

\section{Vascular Access}

Although central venous catheterization by the landmark technique has been used traditionally with success,

From:

Multidisciplinary Intensive Care Unit, Manipal Hospital, Bengaluru,

Karnataka, India

\section{Correspondence:}

Dr. Jose Chacko, Multidisciplinary Intensive Care Unit, Manipal Hospital, Bengaluru, Karnataka, India. E-mail: chackojose@gmail.com difficulties may arise in the presence of anatomical abnormalities or if the vein is thrombosed. Ultrasound guidance for central venous catheterization has been successfully employed for over three decades. ${ }^{[1]}$ Ultrasound may be used to "prescan" the chosen site followed by blind insertion at the location identified, or by real-time guidance and insertion. It may be carried out as a two-person procedure with the probe controlled by a second person, or by a single operator controlling the probe with one hand, while the introducer needle is inserted with the other. It is probably ideal to carry out line insertions under the real-time guidance with a single operator controlling the probe and the introducer needle. A high frequency microconvex or linear probe $(8-15 \mathrm{MHz})$ is best suited for ultrasound guided insertion of lines. Probe sterility may be ensured by using a glove or tegaderm to cover the probe head and a sterile sleeve around the cable that extends up to the connection port on the machine.

\section{Internal jugular vein cannulation}

The internal jugular vein (IJV) is easier to cannulate in the short axis view, especially for beginners. ${ }^{[2]}$ The shaft of 
the needle is difficult to visualize with this technique; the needle tip indents the wall of the vein as it makes contact with it [Figure 1]. Once flashback of blood is obtained, the guidewire is inserted, followed by confirmation of wire position within the vein. A medial-oblique, "in plane" technique allows visualization of the whole length of the needle; besides, the needle tip may be tracked from the point of entry on the skin to the vein. The carotid artery can also be viewed beside the vein, which might help avoid arterial puncture. ${ }^{[3]}$ There is sound evidence to suggest that ultrasound guidance improves the success rate of IJV cannulation. ${ }^{[4-6]}$ Besides reducing access time and number of attempts for successful cannulation, real-time ultrasound guidance has also been shown to reduce the incidence of complications, including carotid puncture, hematoma, hemothorax, pneumothorax and central venous catheter related bloodstream infections. ${ }^{[5]}$

\section{Subclavian vein cannulation}

The value of real-time ultrasound guidance for subclavian vein cannulation is less well-defined than for IJV cannulation. An 8-10 MHz microconvex probe is ideal; a linear high frequency probe would also suffice. The subclavian artery and the vein are initially viewed on the short axis, with the probe marker pointing to the head. The probe is then turned anti-clockwise with the probe marker pointing to the right side of the patient. This brings the vein to view on the long axis [Figure 2]. The vein may be differentiated from the artery by the absence of pulsations, compressibility, variation in size with respiration and using pulse wave Doppler. The pleural slide may be seen deep to the vein. The introducer needle is inserted at the lateral edge of the probe using an "in plane" technique. The needle tip is followed from the skin level, all the way down to the wall of the vein. Indentation of the wall of the vein is seen as the needle tip makes contact with it; at this stage, the needle is advanced into the vein and visualized within the lumen of the vein. It is important to follow the course of the needle throughout to prevent injury to surrounding structures such as the subclavian artery and the pleura. The point of entry is often more lateral compared to the blind technique as the medial part of
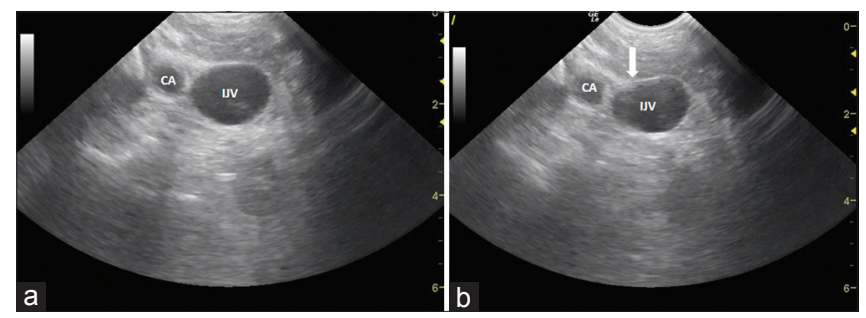

Figure I: Cannulation of the IJV. (a) Short axis view. (b) Indentation of the vein by the needle tip (arrow) (CA: Common carotid artery, IJV: Internal jugular vein) the subclavian vein lies under the clavicle, making it inaccessible to ultrasound imaging. In a randomized controlled trial that compared blind versus real-time ultrasound guided subclavian vein cannulation using the "in plane" technique, catheterization was successful in $100 \%$ of cases compared to $87.5 \%$ with the landmark technique; complications including arterial puncture, hematomas, pneumothorax, hemothorax, phrenic nerve injury, and cardiac tamponade were all significantly more with the landmark technique. ${ }^{[7]}$

\section{Femoral vein cannulation}

Perhaps due to the inherent safety of blind techniques, ultrasound guided femoral venous cannulation has been less studied. Cannulation may be performed in the long or short axis view, depending on operator preference. A long axis view allows visualization of the whole length of the needle and allows tracking the needle tip all the way down to the lumen of the vein. Ultrasound guided femoral vein cannulation has been shown to be useful during cardiopulmonary resuscitation, ${ }^{[8]}$ for insertion of dialysis catheters ${ }^{[0]}$ and in pediatric patients. ${ }^{[10]}$

\section{Deep Vein Thrombosis}

Compression ultrasonography is a well-validated tool for the diagnosis of lower limb DVTs with high levels of specificity and sensitivity. ${ }^{[11,12]}$ Detailed examination of the entire lower limb by a radiologist or technician may be time-consuming and unsuited to emergency settings. In comparison, a modified two-point compression may be expeditiously performed by a trained bedside clinician with equally good diagnostic efficacy. A linear probe of 5-12 MHz frequency is suitable for compression ultrasonography of the lower limb. In the supine position, with the leg externally rotated, the probe is initially applied just distal to the inguinal ligament with the probe marker pointing to the right of the patient. By sliding the probe up and down, the junction of the common femoral vein and the saphenous vein is identified. The common femoral artery is seen immediately lateral to the vein. Firm pressure is applied, until the artery gets mildly

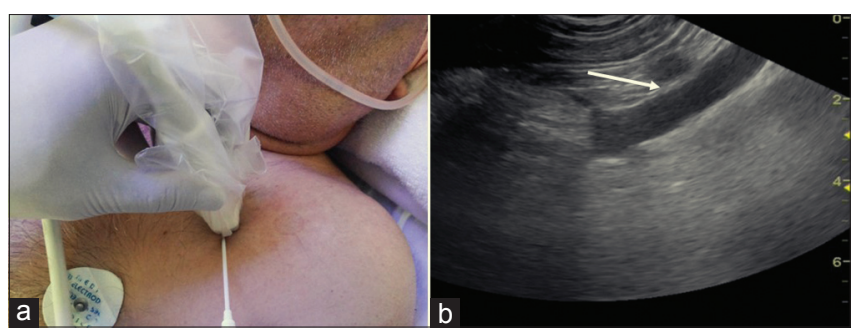

Figure 2: Cannulation of the subclavian vein. (a) The probe is positioned to view the vein in the long axis. Point of entry is at the lateral edge of the probe. (b) Long axis view of the vein, showing the point of needle entry (arrow) 
deformed - if the vein compresses completely with approximation of its walls, a clot can be ruled out. Lack of compressibility indicates a clot within the vein [Figure 3]. A segment of the common femoral vein is examined, $2 \mathrm{~cm}$ proximal and distal to the saphenofemoral junction. Distally, the bifurcation of the common femoral vein to the superficial and deep femoral veins is identified and a clot is excluded by compression. To examine the popliteal vein (PV), the knee is flexed, and the probe is applied to the popliteal fossa, with the probe marker pointing to the patient's right. By sliding the probe up and down, the popliteal artery and PV are identified, with the vein usually located posterior to the artery [Figure 4]. A $2 \mathrm{~cm}$ length of the vein is examined until its division into the superficial and deep tibial veins and the peroneal vein. Veins are best viewed on the transverse (short axis) view. On a sagittal view, the probe may easily slide off the vein when applying pressure, leading to a false impression of compressibility. Although clots may be evident as hyperechoic areas within the lumen of the vein, absence of complete compressibility may be the only sign of a clot, especially if acute. Using color flow Doppler, a clot may be identified as a filling defect; besides, squeezing the calf might help augment venous flow and fill the lumen to demonstrate the absence of a clot. However, the use of color Doppler may not improve the diagnostic sensitivity of DVT. ${ }^{[13]}$ Tomkowski et al. compared the efficacy of compression ultrasonography to diagnose DVT with ascending venography 160 acutely ill medical patients. Compression ultrasonography had a strong negative predictive value of $98 \%$ (95\% confidence interval $[\mathrm{CI}]=95-99 \%)$, but underestimated the incidence of distal DVT with a positive predictive value of $75 \%(\mathrm{CI}=30-95 \%) .{ }^{[14]}$

\section{Abdominal Ultrasound}

\section{Focused assessment with sonography in trauma}

In major trauma, it is crucial to identify the patient who may be bleeding into the peritoneum following blunt abdominal injuries. Although computed tomography (CT) scan is very reliable, transferring a bleeding

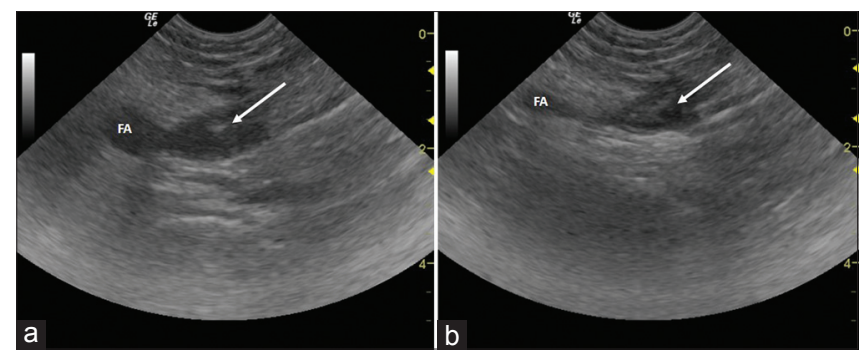

Figure 3: Thrombus in the femoral vein. (a) Hyperechoic shadow of a thrombus within the femoral vein (arrow). (b) Compression causes an indentation of the artery, but the walls of the vein do not collapse because of the thrombus within the lumen (FA: Femoral artery) patient out of the emergency room may be hazardous. Diagnostic peritoneal lavage has been used in this situation; however, it may be oversensitive leading to negative laparotomies. ${ }^{[15]}$ Besides, serial examinations to check for ongoing bleed is not possible.

Focused assessment with sonography in trauma (FAST) surveys the peritoneal and pericardial cavity for free fluid through standard views with the subject lying in the supine position. A curvilinear phased array probe of $3.5-5 \mathrm{MHz}$ frequency is ideally suited for the FAST examination. On the subcostal view, the probe is held horizontally and pressed down to view the heart. Any pericardial collection is noted. For the right upper quadrant view, the probe is held along the thoracoabdominal line and the interface between the liver and the kidney is surveyed for any free fluid in the Morrison's pouch [Figure 5a]. The left upper quadrant is viewed similarly by placing the probe lateral to the spleen along the posterior axillary line to examine the splenorenal pouch. To view the pelvis, the transducer is placed transversely, just above the pubic symphysis to view the bladder. The pouch of Douglas in the female and the rectovesical pouch in the male [Figure 5b] are examined carefully for the presence of free fluid. If free fluid is seen, further imaging of the right and left lower quadrants and the paracolic gutters are undertaken to quantify the volume of fluid. An extended FAST examination may be performed to include examination of the hemithorax bilaterally for evidence of hemothorax and the anterior chest for pneumothorax.${ }^{[16]}$ The FAST examination is a sensitive tool and a useful alternative to CT scans in the unstable patient. It is quick, noninvasive and does not require transfer out of the emergency room; besides, repeat examinations can be performed as appropriate. The specificity and accuracy of FAST has been reported to be $98-100 \% \cdot{ }^{[17,18]}$

\section{Aorta, liver, gall bladder and kidneys}

The abdominal aorta can be assessed in the patient suspected to have an aneurysm by visualizing the entire length of the aorta. A diameter of $<3 \mathrm{~cm}$ all along its path

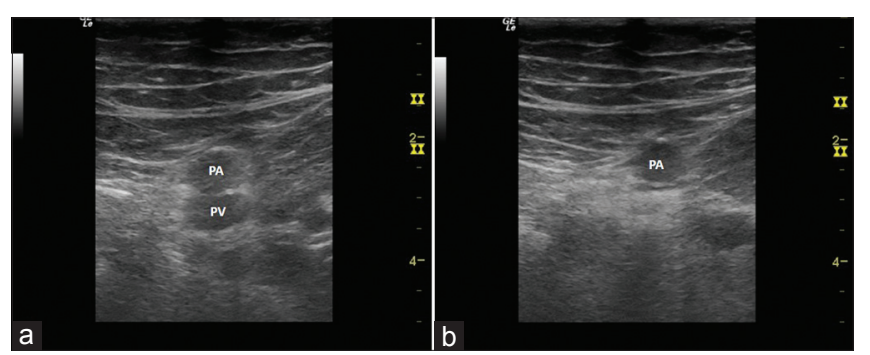

Figure 4: (a) Popliteal artery (PA) and vein (PV). (b) Compression results in complete collapse of the vein indicating patency. The artery does not collapse 
would help to exclude an aneurysm. ${ }^{[19]}$ If the examination is suspicious, further investigations like CT scan may be carried out. Bowel gas may be an impediment to the adequacy of viewing of the aorta. In acute kidney injury, ultrasound may be employed to exclude an obstructive cause. The renal resistive index may help to predict acute kidney injury and to prognosticate outcome. ${ }^{[20]}$ The liver and the gallbladder are well-visualized on the ultrasound scan. A gallbladder wall thickness of $>3 \mathrm{~mm}$ may suggest acute cholecystitis; although, it represents a nonspecific sign that occurs in many different disease processes. ${ }^{[21]}$ The normal diameter of the common bile duct is $<4 \mathrm{~mm}$; a diameter of $>7 \mathrm{~mm}$ may suggest biliary obstruction. ${ }^{[22]}$

\section{Neurological Examination}

It may be important to monitor intracranial pressure (ICP), especially in traumatic brain injury, to detect any rise and direct appropriate therapeutic intervention. Conventionally, ICP is measured invasively by intra-parenchymal or intra-ventricular catheter insertion. Measurement of the optic nerve sheath diameter (ONSD) by ocular ultrasonography may be a useful tool to measure ICP at the bedside [Figure 6]. A high frequency $(7.5 \mathrm{MHz})$ linear probe is applied over the closed upper eyelid with the optic nerve sheath perpendicular to the scanning axis. The optic nerve sheath is measured $3 \mathrm{~mm}$ posterior to the globe. In adults with traumatic brain injury, an ONSD diameter cut-off value of $5.7 \mathrm{~mm}$ predicted raised ICP of $20 \mathrm{~mm} \mathrm{Hg}$ or more. ${ }^{[23]}$ In another prospective study, a single-blinded operator measured ONSD in 27 adult patients with traumatic brain injury and compared it with invasive ICP measurements. A cut-off of $5.2 \mathrm{~mm}$ had a sensitivity of $83 \%(95 \%$ $\mathrm{CI}=35.9-99.6 \%)$ and specificity of $100 \%(95 \% \mathrm{CI}=83.9-$ $100 \%)$ to predict ICP rise of $>20 \mathrm{~mm} \mathrm{Hg.}{ }^{[24]}$

Transcranial Doppler ultrasonography (TCD) may be used to diagnose brain death. ${ }^{[25]}$ TCD is performed using a low frequency probe - a 2-4 MHz echocardiography probe is suitable. The middle cerebral artery is most often studied; insonation is through the temporal window. The probe is placed about $2 \mathrm{~cm}$ above a line joining the

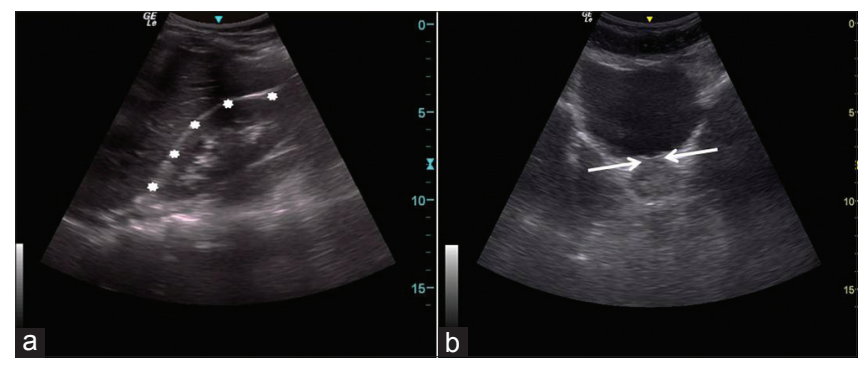

Figure 5: Focused assessment with sonography in trauma scan. (a) Morrison's pouch (asterisks). (b) Rectovesical pouch (arrows) tragus to the lateral canthus of the eye. The probe is placed at right angles to the skull with the probe marker pointing anteriorly. The middle cerebral artery signal is found at a depth of around $5 \mathrm{~cm} \cdot{ }^{[2]}$ Once a signal is obtained, pulse wave Doppler is applied to measure velocity of flow. In cerebral circulatory arrest, typical flow patterns are seen. Diastolic flow ceases when the ICP rises above the diastolic pressure; with a higher $\mathrm{ICP}$, the flow reverses during diastole, resulting in an oscillating flow. With further rise, a "systolic spike" pattern is seen TCD can precisely assess delayed cerebral vasospasm following subarachnoid hemorrhage by measurement of middle cerebral artery blood flow. A mean flow velocity in the middle cerebral artery of $120-150 \mathrm{~cm} / \mathrm{s}$ indicates mild, $151-200 \mathrm{~cm} / \mathrm{s}$ indicates moderate, and $>200 \mathrm{~cm} / \mathrm{s}$ indicates severe vasospasm. ${ }^{[27]}$ One of the main drawbacks of TCD is inability to find a window, especially in women and the elderly.

In patients who have undergone decompressive craniectomy, the brain may be easily visualized through the defect on the skull. ${ }^{[28]}$ The midline can be located for possible shift; hemorrhages and infarcts may also be identified. It may also be possible to measure the width of the ventricles and see the tip of an intra-ventricular catheter. A good agreement has been found between ultrasound and CT images. ${ }^{[28]}$

Extensive orbital edema might prevent examination of the papillary light reflex due to difficulty in retracting swollen eyelids. In this situation, it is relatively simple to assess pupillary size and reaction with ultrasound. A high frequency $(10-13 \mathrm{MHz})$ liner probe is gently placed transversely on the closed eyelid with the probe angled upwards to view the iris and the pupil. A light is shone on the ipsilateral, closed eyelid; the pupillary reaction can be readily appreciated and size measured. ${ }^{[29]}$

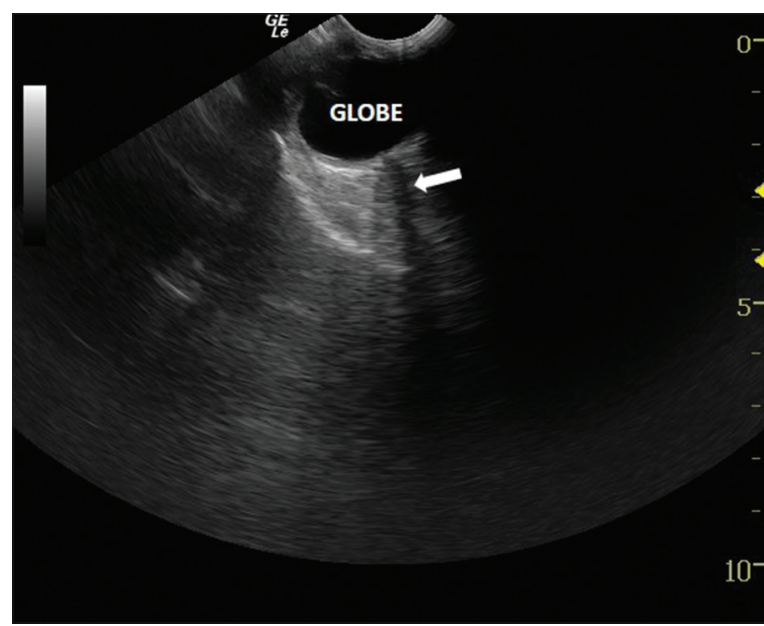

Figure 6: Optic nerve sheath (arrow) 


\section{Ultrasonography of the Airway}

Ultrasound scanning allows direct visualization of lung expansion bilaterally following endotracheal intubation by observing the pleural slide. The degree of displacement of the liver and spleen during a spontaneous breathing trial indicates diaphragmatic movement and may predict successful extubation. ${ }^{[30]}$ An air column width of $<0.8 \mathrm{~mm}$ measured during endotracheal tube cuff deflation may predict postextubation stridor. ${ }^{[31]}$ During percutaneous dilatational tracheostomy (PDT), prescanning of the neck may be performed to check for prominent blood vessels that may be encountered during the procedure. ${ }^{[32]}$ The endotracheal tube may be withdrawn to the level of the cricoid cartilage under ultrasound guidance prior to PDT. ${ }^{[33]}$ The introducer needle and guidewire may be precisely inserted at the appropriate interspace on the trachea, in the midline, using real-time ultrasound guidance and possibly obviate the need for routine bronchoscope use. ${ }^{[34]}$

\section{Conclusions}

The traditional approach to ultrasonographic investigation involves referral to radiology or cardiology as appropriate; a technician performs the imaging followed by interpretation by a specialist with reports being relayed back to the referring physician. Clearly this pathway is unacceptably circuitous in the critically ill-patient when crucial clinical decisions need to be made swiftly, based on physiological alterations. Besides, repeated examinations are often required to assess response to therapeutic interventions. There are imaging techniques unique to critical care, such as ultrasound imaging of the lung, described and utilized almost exclusively by intensivists, prompted by the need to seek quick answers in patients who are too unstable to be transferred out of the intensive care unit. The utility of point of care ultrasonography performed by intensivists is well-established, although caution needs to be exercised regarding likely harm from inappropriate therapeutic decisions made by inadequately trained physicians. Training and curriculum for the practice of critical care ultrasonography needs to be standardized and appropriate credentialing needs to be established. Ultrasonography is being increasingly employed to assist bedside clinical examination and might well turn out to be the stethoscope of the future.

\section{References}

1. Legler D, Nugent M. Doppler localization of the internal jugular vein facilitates central venous cannulation. Anesthesiology 1984;60:481-2.

2. Blaivas M, Brannam L, Fernandez E. Short-axis versus long-axis approaches for teaching ultrasound-guided vascular access on a new inanimate model. Acad Emerg Med 2003;10:1307-11.
3. Dilisio R, Mittnacht AJ. The "medial-oblique" approach to ultrasound-guided central venous cannulation-Maximize the view, minimize the risk. J Cardiothorac Vasc Anesth 2012;26:982-4.

4. Leung J, Duffy M, Finckh A. Real-time ultrasonographically-guided internal jugular vein catheterization in the emergency department increases success rates and reduces complications: A randomized, prospective study. Ann Emerg Med 2006;48:540-7.

5. Karakitsos D, Labropoulos N, De Groot E, Patrianakos AP, Kouraklis G, Poularas J, et al. Real-time ultrasound-guided catheterisation of the internal jugular vein: A prospective comparison with the landmark technique in critical care patients. Crit Care 2006;10:R162.

6. Sigaut S, Skhiri A, Stany I, Golmar J, Nivoche Y, Constant I, et al. Ultrasound guided internal jugular vein access in children and infant: A meta-analysis of published studies. Paediatr Anaesth 2009;19:1199-206

7. Fragou M, Gravvanis A, Dimitriou V, Papalois A, Kouraklis G, Karabinis A, et al. Real-time ultrasound-guided subclavian vein cannulation versus the landmark method in critical care patients: A prospective randomized study. Crit Care Med 2011;39:1607-12.

8. Hilty WM, Hudson PA, Levitt MA, Hall JB. Real-time ultrasound-guided femoral vein catheterization during cardiopulmonary resuscitation. Ann Emerg Med 1997;29:331-6.

9. Kwon TH, Kim YL, Cho DK. Ultrasound-guided cannulation of the femoral vein for acute haemodialysis access. Nephrol Dial Transplant 1997;12:1009-12.

10. Aouad MT, Kanazi GE, Abdallah FW, Moukaddem FH, Turbay MJ, Obeid MY, et al. Femoral vein cannulation performed by residents: A comparison between ultrasound-guided and landmark technique in infants and children undergoing cardiac surgery. Anesth Analg 2010;111:724-8.

11. Jacoby J, Cesta M, Axelband J, Melanson S, Heller M, Reed J. Can emergency medicine residents detect acute deep venous thrombosis with a limited, two-site ultrasound examination? J Emerg Med 2007;32:197-200.

12. Crisp JG, Lovato LM, Jang TB. Compression ultrasonography of the lower extremity with portable vascular ultrasonography can accurately detect deep venous thrombosis in the emergency department. Ann Emerg Med 2010;56:601-10.

13. Bernardi E, Camporese G, Büller HR, Siragusa S, Imberti D, Berchio A, et al. Serial 2-point ultrasonography plus D-dimer vs whole-leg color-coded Doppler ultrasonography for diagnosing suspected symptomatic deep vein thrombosis: A randomized controlled trial. JAMA 2008;300:1653-9

14. Tomkowski WZ, Davidson BL, Wisniewska J, Malek G, Kober J, Kuca P, et al. Accuracy of compression ultrasound in screening for deep venous thrombosis in acutely ill medical patients. Thromb Haemost 2007;97:191-4

15. Jansen JO, Logie JR. Diagnostic peritoneal lavage-An obituary. Br J Surg 2005;92:517-8.

16. Kirkpatrick AW, Sirois M, Laupland KB, Liu D, Rowan K, Ball CG, et al. Hand-held thoracic sonography for detecting post-traumatic pneumothoraces: The Extended Focused Assessment with Sonography for Trauma (EFAST). J Trauma 2004;57:288-95.

17. Rose JS, Levitt MA, Porter J, Hutson A, Greenholtz J, Nobay F, et al. Does the presence of ultrasound really affect computed tomographic scan use? A prospective randomized trial of ultrasound in trauma. J Trauma 2001;51:545-50.

18. McKenney M, Lentz K, Nunez D, Sosa JL, Sleeman D, Axelrad A, et al. Can ultrasound replace diagnostic peritoneal lavage in the assessment of blunt trauma? J Trauma 1994;37:439-41.

19. Wilmink AB, Forshaw M, Quick CR, Hubbard CS, Day NE. Accuracy of serial screening for abdominal aortic aneurysms by ultrasound. J Med Screen 2002;9:125-7.

20. Deruddre S, Cheisson G, Mazoit JX, Vicaut E, Benhamou D, Duranteau J. Renal arterial resistance in septic shock: Effects of increasing mean arterial pressure with norepinephrine on the renal resistive index assessed with Doppler ultrasonography. Intensive Care Med 2007;33:1557-62.

21. Handler S.J. Ultrasound of gallbladder wall thickening and its relation to cholecystitis. AJR Am J Roentgenol 1979;132:581-5. 
22. Parulekar SG. Ultrasound evaluation of common bile duct size. Radiology 1979;133:703-7.

23. Soldatos T, Karakitsos D, Chatzimichail K, Papathanasiou M, Gouliamos A, Karabinis A. Optic nerve sonography in the diagnostic evaluation of adult brain injury. Crit Care 2008;12:R67.

24. Frumin E, Schlang J, Wiechmann W, Hata S, Rosen S, Anderson C, et al. Prospective analysis of single operator sonographic optic nerve sheath diameter measurement for diagnosis of elevated intracranial pressure. West J Emerg Med 2014;15:217-20.

25. Feri M, Ralli L, Felici M, Vanni D, Capria V. Transcranial Doppler and brain death diagnosis. Crit Care Med 1994;22:1120-6.

26. Santalucia P, Feldmann E. The basic transcranial Doppler examination: Technique and anatomy. In: Babikian V, Wechsler LR, editors. Transcranial Doppler Ultrasonography. Boston: Butterworth-Heinemann; 1999. p. 13-32.

27. Kassab MY, Majid A, Farooq MU, Azhary H, Hershey LA, Bednarczyk EM, et al. Transcranial Doppler: An introduction for primary care physicians. J Am Board Fam Med 2007;20:65-71.

28. Rincon F. Bedside transcranial sonography: A promising tool for the neurointensivist. Crit Care Med 2012;40:1969-70.

29. Harries A, Shah S, Teismann N, Price D, Nagdev A. Ultrasound assessment of extraocular movements and pupillary light reflex in ocular trauma. Am J Emerg Med 2010;28:956-9.

30. Kim WY, Suh HJ, Hong SB, Koh Y, Lim CM. Diaphragm dysfunction assessed by ultrasonography: Influence on weaning from mechanical ventilation. Crit Care Med 2011;39:2627-30.

31. Ding LW, Wang HC, Wu HD, Chang CJ, Yang PC. Laryngeal ultrasound: A useful method in predicting post-extubation stridor A pilot study. Eur Respir J 2006;27:384-9.

32. Flint AC, Midde R, Rao VA, Lasman TE, Ho PT. Bedside ultrasound screening for pretracheal vascular structures mav minimize the risks of percutaneous dilatational tracheostomy. Neurocrit Care 2009;11:372-6.

33. Rodríguez SJ, Esteves LE. Real-time ultrasound-guided percutaneous dilatational tracheostomy. Crit Care 2011;15:443.

34. Chacko J, Nikahat J, Gagan B, Umesh K, Ramanathan M. Real-time ultrasound-guided percutaneous dilatational tracheostomy. Intensive Care Med 2012;38:920-1.

How to cite this article: Chacko J! , Brar G. Bedside ultrasonography-Applications in critical care: Part II. Indian J Crit Care Med 2014;18:376-81.

Source of Support: Nil, Conflict of Interest: None declared. 